Service social

\title{
Détresse et anxiété chez les étudiants du collégial et recours aux services d'aide socioaffectifs
}

\section{Marc-André Gosselin et Robert Ducharme}

Volume 63, numéro 1, 2017

Interventions en matière d'agressions sexuelles

URI : https://id.erudit.org/iderudit/1040048ar

DOI : https://doi.org/10.7202/1040048ar

Aller au sommaire du numéro

\section{Éditeur(s)}

École de service social de l’Université Laval

ISSN

1708-1734 (numérique)

Découvrir la revue

Citer cet article

Gosselin, M.-A. \& Ducharme, R. (2017). Détresse et anxiété chez les étudiants du collégial et recours aux services d'aide socioaffectifs. Service social, 63(1), 92-104. https://doi.org/10.7202/1040048ar
Résumé de l'article

Cette étude a été réalisée auprès de 12208 cégépiens du régulier, inscrits à la session d'automne 2014 et provenant de huit cégeps situés dans différentes régions du Québec. Elle mesure l'ampleur des besoins socioaffectifs des répondants et l'utilisation des services d'aide qui leur sont offerts. Les résultats indiquent que les trois quarts des répondants ont recours à de tels services d'aide pour des problèmes autres que scolaires. Les principaux motifs de ce recours sont la satisfaction, la curiosité et la souffrance. Les résultats montrent aussi que $17,4 \%$ des répondants ont ressenti beaucoup ou énormément de détresse et que 35,1\% ont éprouvé souvent ou tout le temps de l'anxiété. Les principaux facteurs explicatifs de la détresse et de l'anxiété sont les conflits familiaux, la pression liée au rendement scolaire, l'intimidation et les pensées suicidaires. Ainsi, 29,3\% disent qu'ils ont vécu beaucoup ou énormément de détresse à l'égard des conflits familiaux; 33,3 \% ont éprouvé beaucoup ou énormément de pression liée à la performance scolaire; $11,9 \%$ ont ressenti beaucoup ou énormément de détresse liée à l'intimidation et 18,3\% des répondants affirment avoir eu des pensées suicidaires et parmi eux, 7,3\% disent en avoir eues souvent ou tout le temps. Enfin, certains préjugés à l'égard de la santé mentale apparaissent comme un frein significatif à l'utilisation des ressources d'aide.
Ce document est protégé par la loi sur le droit d'auteur. L'utilisation des services d'Érudit (y compris la reproduction) est assujettie à sa politique d'utilisation que vous pouvez consulter en ligne.

https://apropos.erudit.org/fr/usagers/politique-dutilisation/ 


\title{
Détresse et anxiété chez les étudiants du collégial et recours aux services d'aide socioaffectifs
}

\author{
GOSSELIN, Marc-André \\ DUCHARME, Robert
}

\section{RÉSUMÉ}

Cette étude a été réalisée auprès de 12208 cégépiens du régulier, inscrits à la session d'automne 2014 et provenant de huit cégeps situés dans différentes régions du Québec. Elle mesure l'ampleur des besoins socioaffectifs des répondants et l'utilisation des services d'aide qui leur sont offerts. Les résultats indiquent que les trois quarts des répondants ont recours à de tels services d'aide pour des problèmes autres que scolaires. Les principaux motifs de ce recours sont la satisfaction, la curiosité et la souffrance. Les résultats montrent aussi que $17,4 \%$ des répondants ont ressenti beaucoup ou énormément de détresse et que $35,1 \%$ ont éprouvé souvent ou tout le temps de l'anxiété. Les principaux facteurs explicatifs de la détresse et de l'anxiété sont les conflits familiaux, la pression liée au rendement scolaire, l'intimidation et les pensées suicidaires. Ainsi, 29,3\% disent qu'ils ont vécu beaucoup ou énormément de détresse à l'égard des conflits familiaux; 33,3\% ont éprouvé beaucoup ou énormément de pression liée à la performance scolaire; 11,9\% ont ressenti beaucoup ou énormément de détresse liée à l'intimidation et 18,3\% des répondants affirment avoir eu des pensées suicidaires et parmi eux, 7,3\% disent en avoir eues souvent ou tout le temps. Enfin, certains préjugés à l'égard de la santé mentale apparaissent comme un frein significatif à l'utilisation des ressources d'aide.

Mots clés : détresse, anxiété, suicide, intimidation, conflits parentaux, pression liée à la performance scolaire, ressources d'aide psychosociales, groupes ethniques, préjugés, santé mentale.

\begin{abstract}
This study measures the state of distress and anxiety among 12,208 college students, registered for the 2014 fall semester as well as their level of prejudice towards mental health and the use of various psychosocial support services. The study highlights the importance of providing student support services who are experiencing personal difficulties while highlighting family conflict, the pressure of school performance, bullying and suicidal thoughts as determinants of psychosocial issues. Although the level of prejudice against mental health among respondents is moderately high, there is a significant barrier to utilisation of aid resources. The anterior satisfaction and curiosity are the main reasons given for utilisation with help internal resources; however, suffering is an important reason for utilisation.
\end{abstract}

Keywords: suicidal ideation, mental school health, distress, anxiety, suicide, bullying, parental conflicts, pressure related to performance, psychosocial assistance resources, ethnic groups, prejudice. 


\section{INTRODUCTION ET DÉFINITION DU PROBLÈME}

Les cégeps disposent tous de services pour venir en aide aux élèves qui éprouvent de la détresse psychologique dans leur cheminement scolaire. Cette détresse découle des événements stressants de la vie quotidienne tels que la pression liée à leur réussite scolaire, les conflits familiaux, les ruptures amoureuses, les échecs multiples dans leur vie sociale, voire le rejet, l'intimidation, de même que leur intégration culturelle. Dans ce cas-ci, on parlera de leur situation de détresse en référence aux travaux de Holmes et Raye (1967) sur le sujet.

Dans la même ligne d'idée Lesage, Bernèche et Bordeleau (2010) soulignent que la détresse psychologique est un indicateur important en santé mentale. Ils mentionnent que lorsqu'elle est persistante, elle peut mener, chez les 15 à 24 ans, à des troubles dépressifs et d'anxiété. Ils indiquent, par ailleurs, les principaux signes permettant de voir qu'une personne vit de la détresse dont les symptômes physiques, tels que la perte ou l'augmentation notable du poids, les symptômes cognitifs, tels que les difficultés de concentration, l'indécision, les pensées répétitives de mort ou de suicide, les réactions émotionnelles fortes, telles que la tristesse, l'irritabilité, le sentiment d'être inutile, la perte d'espoir et de plaisir, la fatigue, la culpabilité, et les troubles comportementaux tels que le changement dans l'alimentation et le sommeil, le ralentissement moteur, etc. (Lesage et al., 2010).

Ajoutons à cette liste de symptômes que plusieurs cégépiens et cégépiennes, à cette période critique de leur développement socioaffectif, peuvent avoir tendance à se faire du souci, à anticiper les difficultés et parfois à s'en créer là où il n'y en a pas, de sorte qu'ils peuvent ressentir diverses manifestations liées à l'anxiété dans diverses dimensions de leur personnalité. Beck et Steer (1988) parlent, en ce sens, de divers symptômes physiques telles que la tension musculaire, les tremblements, les étourdissements, la sudation, les engourdissements, la douleur à la poitrine, les palpitations cardiaques comme autant de signes majeurs d'anxiété. Ils ajoutent à cette liste divers symptômes cognitifs tels que l'hyper vigilance, la confusion, la difficulté à se concentrer et à contrôler ses pensées, les oublis, les pensées suicidaires, les distorsions cognitives de même que des symptômes émotionnels tels que la peur, la nervosité, l'irritabilité et l'impatience, et des symptômes comportementaux tels que l'inhibition, l'évitement, l'agitation, les discours incohérents, etc.) pour tracer un portrait assez complet des manifestations d'anxiété. Dans ce cas, ils reprennent à leur compte la définition de l'Association américaine de psychiatrie sur le concept d'anxiété (APA, 2013).

Selon Villatte, Marcotte et Potvin (2015), qui citent les travaux de Daddona (2011) et de Fier et Brzezinski (2010), le nombre d'étudiants de niveaux supérieurs souffrant de problèmes de santé mentale serait en très forte hausse. Cette augmentation, selon elles, « fait l'objet d'une préoccupation importante tant chez les psychologues scolaires que chez les enseignants, non seulement parce qu'elle met en lumière la nécessité d'un nombre croissant de ressources, mais aussi d'un changement d'orientation dans les types de services à offrir ».

Par ailleurs, à propos des ressources d'aide dans les cégeps, il appert que, même s'ils disposent tous de services pour venir en aide aux élèves qui éprouvent de telles difficultés personnelles, il existe de très grandes disparités entre les établissements quant à la nature des services offerts et surtout quant à leur fréquentation (Gosselin et Ducharme, 2015). Ainsi, dans certains cégeps, on dispose de psychologues à temps plein, alors que dans plusieurs autres, on n'en a tout simplement pas ou on en a à temps partiel. Dans d'autres cégeps, on dispose de travailleurs sociaux, alors que, dans la majorité des autres cégeps, il n'y en pas du tout. II en est de même pour les infirmières, les éducateurs et 
éducatrices spécialisés, les intervenants en relation d'aide et les services d'aide téléphonique. Dans les cas où de tels services ne sont pas disponibles, ce sont souvent les professeurs, les aides pédagogiques individuels (API) et les conseiller en orientation (CO) qui doivent suppléer à ce manque de ressources internes (Gosselin et Ducharme, 2015) ou tout bonnement les ressources professionnelles disponibles dans la communauté externe du cégep.

\section{Contexte}

L'enquête fait suite au désir des cégeps de connaître les besoins socioaffectifs des étudiants et leur recours aux services d'aide. Un premier sondage avait été réalisé au collège de Bois-de-Boulogne à l'automne 2013 et les résultats ont incité huit cégeps à reproduire cette enquête à l'automne 2014. Le présent article fait état des résultats de cette dernière enquête.

\section{Objectifs et questions de recherche}

Cette étude vise à mesurer l'utilisation des services d'aide ainsi qu'à identifier les facteurs qui facilitent ou qui freinent le processus du recours aux services d'aide chez les étudiants du réseau collégial. À partir de cet objectif général, les hypothèses suivantes ont été explorées:

- Les étudiants et les étudiantes utilisent les ressources d'aide.

- Il existe une différence selon le sexe et l'âge dans la fréquentation des services d'aide, dans les motifs d'utilisation, dans les niveaux de détresse et d'anxiété ainsi que dans les préjugés et attitudes envers la santé mentale.

- Les niveaux élevés de détresse et d'anxiété indiquent que les étudiants et étudiantes des cégeps ont besoin de services socioaffectifs.

- Les préjugés en santé mentale nuisent à l'utilisation des services d'aide socioaffectifs.

\section{MÉTHODOLOGIE}

La stratégie utilisée est un questionnaire en ligne sur la plateforme "Omnivox » de chacun des collèges participants. Un message des chercheurs invitait les étudiants à participer au sondage sur une base libre et volontaire lorsqu'ils accédaient au portail du cégep. Le questionnaire était disponible durant une période de quinze jours à partir du 15 novembre 2014 et garantissait l'anonymat et la confidentialité des réponses.

Huit cégeps ont participé à cette enquête dont un collège de Montréal, deux de Québec et cinq des régions périphériques. En tout, 12208 étudiants ont dûment complété le questionnaire. Le pourcentage de participation des répondants par collège varie de $33,1 \%$ à $57,3 \%$. Les filles représentent $65,3 \%$ de l'échantillon et les garçons $34,7 \%$ ce qui reflète à $4 \%$ près leurs taux respectifs de fréquentation scolaire dans les cégeps participants. L'âge des répondants représente fidèlement la répartition des étudiants du collégial avec un contingent de $80 \%$ dans la catégorie des 17 à 20 ans.

L'outil intitulé "Les besoins psychosociaux des étudiants et la consultation des services d'aide dans le réseau collégial " a été développé à partir de l' "Échelle de changement de vie de Holmes et Rahe " (1967), adaptée pour les étudiants de niveau collégial et de l' «Inventaire d'anxiété de Beck (1992) ». II comporte neuf questions réparties en quatre sections. La première section porte sur des données sociodémographiques telles que le cégep fréquenté, le sexe, l'âge, le groupe ethnique, l'année d'étude 
ainsi que le programme d'études. La deuxième section traite du recours aux services d'aide à l'interne, de leurs motifs ainsi que de l'utilisation des services d'aide à l'externe. La troisième section porte sur la mesure de la détresse qui est composée de 22 items et de l'anxiété qui en contient 21. Ces composantes apparaissent aux tableaux 2 et 3 qui suivent. Enfin, la quatrième section aborde les préjugés à l'égard de la santé mentale dont les 19 caractéristiques figurent au tableau 4.

Trois types de tests statistiques ont été utilisés dans le traitement des données : des Tests $t$ de Student, des corrélations et des régressions linéaires. Seuls les résultats qui étaient minimalement significatifs à $\mathrm{p}<.01$ ont été retenus. Le logiciel utilisé pour traiter les données est la version 22.0 de SPSS.

\section{RÉSULTATS}

À la question portant sur l'utilisation des ressources d'aide à l'interne, 72,9 \% des répondants disent les avoir consultées pour des difficultés personnelles autres que celles liées à la dimension scolaire. Le tableau 1 présente la répartition des ressources d'aide selon leur importance de fréquentation.

Tableau 1 Pourcentage de fréquentation des ressources d'aide au cégep

\begin{tabular}{|c|c|c|c|c|c|}
\hline & & Total & Filles & Garçons & Test $t$ \\
\hline 1 & Professeur & 58,4 & 61,7 & 52,1 & $\mathrm{p}<.001$ \\
\hline 2 & Aide pédagogique (API) & 51,0 & 53,7 & 45,8 & $\mathrm{p}<.000$ \\
\hline 3 & Conseiller d'orientation (CO) & 24,0 & 25,7 & 20,9 & $\mathrm{p}<.000$ \\
\hline 4 & Agent de sécurité & 14,4 & 14,5 & 14,2 & $\mathrm{n} . \mathrm{s}$. \\
\hline 5 & Infirmière & 13,6 & 16,0 & 9,0 & $\mathrm{p}<.000$ \\
\hline 6 & Aide spécialisée & 9,2 & 9,7 & 8,2 & $\mathrm{p}<.001$ \\
\hline 7 & Psychologue & 6,2 & 7,0 & 4,5 & $\mathrm{p}<.000$ \\
\hline 8 & Travailleur social & 6,0 & 6,7 & 4,6 & $\mathrm{p}<.000$ \\
\hline 9 & Éducateur spécialisé & 3,5 & 3,6 & 3,3 & $\mathrm{n} . \mathrm{s}$. \\
\hline 10 & Soutien téléphonique & 2,9 & 3,0 & 2,8 & $\mathrm{n} . \mathrm{s}$. \\
\hline 11 & Soutien via portail & 2,2 & 2,0 & 2,6 & $\mathrm{p}<.000$ \\
\hline & Intervenants en relation & & 1,0 & 1,1 & $\mathrm{n} . \mathrm{s}$. \\
\hline 12 & d'aide & 1,1 & & & $\mathrm{n} . \mathrm{s}$. \\
\hline 13 & Centres d'aide & 0,8 & 0,8 & 0,8 & $\mathrm{p}<.000$ \\
\hline 14 & Aide financière & 0,3 & 0,4 & 0,1 & $\mathrm{n} . \mathrm{s}$. \\
\hline 15 & Tutorat par les pairs & 0,3 & 0,4 & 0,3 & $\mathrm{n} . \mathrm{n}$ \\
\hline 16 & Socioculturel & 0,2 & 0,2 & 0,2 & $\mathrm{n} . \mathrm{s}$ \\
\hline 17 & Physio & 0,1 & 0,1 & 0,1 & \\
\hline
\end{tabular}

Les résultats de ce tableau indiquent que les professeurs sont les premières ressources d'aide utilisées pour des problèmes personnels $(58,4 \%)$ suivis par les aides pédagogiques $(51 \%)$ et les conseillers d'orientation (24\%). Par ailleurs, plusieurs répondants ont eu recours aux services d'un agent de sécurité $(14,4 \%)$, à l'infirmière (13,6\%), au service d'aide spécialisée (9,2\%), au psychologue $(6,2 \%)$ et au travailleur social (6,0\%). Les filles se distinguent significativement des garçons sur 8 des 17 ressources d'aide comme l'indique la dernière colonne du tableau 1, tandis que les garçons se distinguent significativement sur une seule ressource, le soutien via portail.

\section{Motifs d'utilisation des ressources d'aide}

Les principales raisons pour lesquelles les étudiants ont eu recours à une ressource d'aide sont : 
- J'avais déjà consulté un service d'aide auparavant et j'avais obtenu des résultats satisfaisants $(24,9 \%)$.

- Je voulais voir par curiosité ce que le service d'aide avait à m'offrir (12,7\%).

- Un professeur m'a référé à un service d'aide $(12,1 \%)$.

- Mon niveau de souffrance était tellement élevé que c'était ma seule issue (8,6\%).

Par ailleurs, peu d'étudiants disent avoir consulté les ressources d'aide suite à la publicité ou à la promotion du service $(4,8 \%)$, suite à une activité organisée par le service $(2,2 \%)$ ou suite à la recommandation d'un parent ou d'un conseiller religieux $(0,2 \%)$. Ces activités ne sont certes pas inutiles, mais elles ont moins d'importance que la satisfaction du service et la curiosité.

\section{Utilisation des ressources d'aide à l'externe}

Le portrait de l'utilisation des ressources d'aide à l'externe est fort différent de celui des ressources internes et présente une plus grande diversité de recours d'aide. Ainsi, $85,1 \%$ des répondants disent avoir utilisé une ressource d'aide à l'externe comparativement 72,9\% à l'interne.

Ce sont les amis qui arrivent au premier rang avec un pourcentage de $72,8 \%$ d'utilisation, suivis des membres de la famille $(72,6 \%)$. Ces deux ressources obtiennent des scores plus élevés que l'utilisation des services professionnels pour lesquels on obtient les résultats suivants : le médecin $(62,5 \%)$, l'infirmière $(36,7 \%)$, le conseiller d'orientation (29\%), le psychologue $(25 \%)$ et le travailleur social $(11,3 \%)$. Par ailleurs, l'utilisation des ressources d'aide spécialisées chez les personnes qui éprouvent de graves problèmes de nature socioaffective obtient un pourcentage non négligeable de 7,9\% dans le cas des sites d'aide en ligne et de 6,4\% concernant les lignes d'écoute téléphonique. Même si à priori les pourcentages ne semblent pas élevés, ils tendent à indiquer un recours d'urgence chez les étudiants en détresse. Finalement, les données indiquent que le recours à d'autres ressources d'aide à l'externe est relativement faible et rejoignent moins de $5 \%$ des répondants.

\section{Détresse}

La détresse est la première variable socioaffective étudiée dans cette enquête. Afin d'évaluer la détresse, une échelle de mesure composée de 22 indicateurs a été créée. Les répondants avaient à indiquer leur niveau de détresse ressentie à l'aide d'un barème variant de "Aucune ", "Peu ", «Beaucoup » à «Énormément ». En attribuant un score de « 0 » pour «Aucune » et de « 3 » pour "Énormément », on obtient une échelle qui varie entre 0 et 66 . La mesure de l'alpha de Cronbach donne .95 pour les 22 éléments qui mesurent notre construit ce qui indique une cohérence interne relativement forte.

La moyenne obtenue à cette variable a été de 14,2 . Plus précisément, $82,6 \%$ des répondants obtiennent un score global indiquant qu'ils n'ont ressenti aucune ou peu de détresse tandis que 17,4\% disent en avoir ressentie beaucoup ou énormément. Par contre, 6,3\% des répondants (770 étudiants) obtiennent un résultat indiquant qu'ils ressentent énormément de détresse à l'égard de plusieurs énoncés comme le montre le tableau 2.

Dans ce tableau, la première colonne indique le pourcentage de ceux qui ont ressenti de la détresse à l'endroit de l'énoncé. Les trois suivantes indiquent la ventilation des réponses selon qu'ils en ont ressenti 
«Peu », «Beaucoup » ou «Énormément» et la dernière colonne présente la somme des «Beaucoup » et «Énormément ».

Tableau 2 Pourcentage de détresse ressentie à l'égard des événements suivants

\begin{tabular}{|c|c|c|c|c|c|}
\hline & Total & Peu & Beaucoup & Énormément & $\begin{array}{c}\text { Somme } \\
\text { (Beaucoup + } \\
\text { Énormément) }\end{array}$ \\
\hline Fatigue, épuisement & 73,6 & 31 & 28,3 & 14,2 & 42,5 \\
\hline Conflit familial & 62,2 & 32,8 & 19,1 & 10,2 & 29,3 \\
\hline $\begin{array}{l}\text { Pression liée à la performance } \\
\text { scolaire }\end{array}$ & 61,4 & 28 & 21,1 & 12,2 & 33,3 \\
\hline Difficulté scolaire & 58,1 & 30,6 & 18,8 & 8,7 & 27,5 \\
\hline Sentiment de solitude & 57,6 & 32,5 & 14,8 & 10,3 & 25,1 \\
\hline Humeur dépressive & 53,3 & 25,4 & 15,4 & 12,5 & 27,9 \\
\hline Problème d'estime personnelle & 50,9 & 26,6 & 13,5 & 10,7 & 24,2 \\
\hline Rupture amoureuse & 48,7 & 20 & 15,9 & 12,8 & 28,7 \\
\hline Mortalité (proche, parent, ami(e)) & 45,4 & 22,8 & 12 & 10,5 & 22,5 \\
\hline Problème financier & 44,1 & 22,7 & 12,3 & 9 & 21,3 \\
\hline Épisode de panique & 39,2 & 17,3 & 10,9 & 11,1 & 22,0 \\
\hline Sautes d'humeur & 34,4 & 18,7 & 7,5 & 8,2 & 15,7 \\
\hline Crise identitaire & 23,1 & 10,4 & 5,1 & 7,7 & 12,8 \\
\hline Difficulté sexuelle & 21,6 & 11,1 & 3,4 & 7,1 & 10,5 \\
\hline Trouble des conduites alimentaires & 20,7 & 9,3 & 3,7 & 7,7 & 11,4 \\
\hline Rejet & 20,4 & 9,5 & 3,5 & 7,4 & 10,9 \\
\hline Victime d'intimidation & 19 & 7,1 & 3,9 & 8 & 11,9 \\
\hline Maladie physique & 18 & 7,4 & 3,6 & 7,1 & 10,7 \\
\hline Agression ou violence à mon égard & 17,1 & 5,7 & 3,3 & 8,1 & 11,4 \\
\hline $\begin{array}{c}\text { Problème de consommation (alcool } \\
\text { et drogue) }\end{array}$ & 15,9 & 6 & 2,8 & 7,2 & 10,0 \\
\hline Conflit culturel & 11,3 & 3,4 & 1,3 & 6,6 & 7,9 \\
\hline Handicap physique & 10,9 & 2,8 & 1,2 & 6,9 & 8,1 \\
\hline
\end{tabular}

À titre d'exemple, $73,6 \%$ des répondants ont ressenti de la détresse à l'égard de leur état de fatigue et d'épuisement, dont $31 \%$ «Peu », 28,3\% «Beaucoup » et 14,2\% «Énormément ». Ainsi, 42,5\% des répondants ont ressenti «Beaucoup » ou «Énormément 》 de détresse à l'endroit de cet énoncé. L'épuisement, les conflits familiaux, la pression liée à la performance scolaire, les difficultés scolaires, le sentiment de solitude et l'humeur dépressive ressortent comme les facteurs les plus fréquents, tandis que le handicap physique, les conflits culturels, le problème de consommation (alcool et drogue) et l'agression ou la violence sont les moins fréquents.

Toutefois, les handicaps physiques, les conflits culturels, les problèmes de consommation ont une incidence moindre mais non négligeable sur la détresse. Par exemple, à peine plus de $10 \%$ disent avoir ressenti de la détresse à cause d'un handicap physique, ce qui pourrait paraître faible mais assurément problématique pour les répondants concernés par cette réalité. Le cas de l'intimidation est un autre exemple qui illustre cette situation. Environ $20 \%$ des répondants ont indiqué avoir ressenti de la 
détresse suite à une situation d'intimidation ou de rejet, ce qui pourrait paraître peu, mais un étudiant sur cinq qui se dit victime d'intimidation ou de rejet au point où cette situation nuisait à ses études et à sa vie sociale ou n'arrivait plus à fonctionner normalement dans toutes les composantes de sa vie, n'est certes pas banal.

\section{Anxiété}

L'anxiété constitue la deuxième variable socioaffective en importance étudiée dans cette enquête. Afin d'évaluer l'anxiété, une échelle de mesure composée de 21 indicateurs a été développée. Les répondants avaient à indiquer la fréquence des symptômes d'anxiété ressentie à l'aide d'un barème variant de « Jamais », «Parfois », «Souvent » et «Tout le temps ». En attribuant un score de « 0 » pour « Jamais » et de « 3 » pour «Tout le temps», on obtient une échelle qui varie entre 0 et 63 . La mesure de l'alpha de Cronbach donne .95 pour les 21 éléments qui mesurent notre construit ce qui indique à nouveau une cohérence interne relativement forte. Le tableau 3 présente le pourcentage des répondants disant avoir ressenti de l'anxiété à l'endroit de chacun des indicateurs de l'échelle d'anxiété.

Tableau $3 \quad$ Anxiété ressentie aux différents énoncés selon le pourcentage

\begin{tabular}{|c|c|c|c|c|c|}
\hline & Total & Parfois & Souvent & $\begin{array}{l}\text { Tout le } \\
\text { temps }\end{array}$ & $\begin{array}{c}\text { Somme } \\
\text { (Souvent } \\
+ \text { Tout le } \\
\text { temps) }\end{array}$ \\
\hline Difficulté à vous endormir & 71,6 & 36 & 22,9 & 12,7 & 35,6 \\
\hline Peur d'échouer & 71 & 32,5 & 21,8 & 16,8 & 38,6 \\
\hline $\begin{array}{l}\text { Impression de ne pas être à la hauteur de } \\
\text { vos idéaux de réussite }\end{array}$ & 69,9 & 29,8 & 23,4 & 16,6 & 40 \\
\hline Doutes quant à votre capacité de réussir & 67,8 & 34,1 & 20,4 & 13,3 & 33,7 \\
\hline Crainte de décevoir & 67,2 & 30,6 & 21,1 & 15,5 & 36,6 \\
\hline Difficulté à vous détendre. & 65,8 & 34,3 & 20,7 & 10,8 & 31,5 \\
\hline $\begin{array}{l}\text { Nervosité ou sensation de tremblements } \\
\text { intérieurs }\end{array}$ & 64,6 & 39,3 & 19,2 & 6 & 25,2 \\
\hline $\begin{array}{c}\text { Tendance à vous faire du souci ou à vous } \\
\text { inquiéter }\end{array}$ & 64,5 & 30 & 21,7 & 12,9 & 34,6 \\
\hline $\begin{array}{l}\text { Tendance à être facilement irritable ou } \\
\text { importuné(e) }\end{array}$ & 62,2 & 36,3 & 18,1 & 7,8 & 25,9 \\
\hline Incapacité à tolérer l'échec & 55,7 & 26,7 & 15,4 & 13,5 & 28,9 \\
\hline Peur d'être jugé(e) & 54,7 & 29,3 & 14,1 & 11,3 & 25,4 \\
\hline $\begin{array}{l}\text { Incapacité à vous libérer de pensées } \\
\text { obsédantes. }\end{array}$ & 53,7 & 28 & 15,9 & 9,8 & 25,7 \\
\hline Nausées, douleurs ou malaises d'estomac & 52,5 & 35,1 & 12,2 & 5,3 & 17,5 \\
\hline $\begin{array}{l}\text { Palpitations ou impression que votre cœur } \\
\text { bat plus fort et plus vite }\end{array}$ & 52 & 33,8 & 13,1 & 5,1 & 18,2 \\
\hline Tendance à sursauter facilement & 46 & 24,3 & 11,5 & 10,3 & 21,8 \\
\hline $\begin{array}{l}\text { Impression de ne pas être aimé(e) pour qui } \\
\text { vous êtes réellement }\end{array}$ & 42 & 23,2 & 10,3 & 8,6 & 18,9 \\
\hline $\begin{array}{c}\text { Sensation de peur comme si quelque chose } \\
\text { d'horrible allait vous arriver }\end{array}$ & 38,6 & 24,3 & 8,9 & 5,4 & 14,3 \\
\hline
\end{tabular}




\begin{tabular}{|c|c|c|c|c|c|}
\hline $\begin{array}{c}\text { Tendance à vous éveiller très tôt le matin et } \\
\text { à rester éveillé(e) }\end{array}$ & 38,5 & 21,1 & 9,3 & 8,2 & 17,5 \\
\hline $\begin{array}{c}\text { Sentiment de nervosité lorsque vous êtes } \\
\text { seul(e) }\end{array}$ & 36,4 & 21 & 8,8 & 6,7 & 15,5 \\
\hline Sensation soudaine de panique & 35,9 & 20,1 & 9 & 6,9 & 15,9 \\
\hline Pensées suicidaires & 18,6 & 11,3 & 2,6 & 4,7 & 7,3 \\
\hline
\end{tabular}

La moyenne obtenue à cette variable a été de 18,5. Presque les deux tiers des répondants (64,9\%) obtiennent un score global indiquant qu'ils n'ont ressenti aucune ou peu d'anxiété tandis que $35,1 \%$ disent en avoir ressentie souvent ou tout le temps. Au total, 6,8 \% (830 étudiants) obtiennent un résultat indiquant qu'ils ressentent tout le temps de l'anxiété liée à de nombreux indicateurs de l'échelle de mesure. Dans ce tableau, la première colonne indique le pourcentage de ceux qui ont ressenti de l'anxiété à l'endroit de l'énoncé. Les trois suivantes indiquent la ventilation des réponses selon qu'ils en ont ressenti «Parfois», "Souvent » ou « Tout le temps » et la dernière colonne présente la somme des "Souvent » et des "Tout le temps ».

Les résultats de ce tableau indiquent que plus des deux tiers des répondants éprouvent de la difficulté à s'endormir, ont peur d'échouer, ont l'impression de ne pas être à la hauteur de leurs idéaux de réussite, ont des doutes quant à leur capacité de réussir et ont peur de décevoir. En conséquence, plus du tiers des répondants éprouvent souvent ou tout le temps de l'anxiété à l'égard des mêmes énoncés.

En contrepartie, la sensation soudaine de panique, le sentiment de nervosité, la tendance à s'éveiller très tôt le matin et la sensation de peur rejoignent un tiers ou plus des répondants et les pensées suicidaires concernent un répondant sur cinq. À ce sujet, l'importance des pensées suicidaires dans le contexte des problèmes socioaffectifs mérite une attention particulière et on y reviendra plus loin dans le texte.

\section{Relation entre la détresse et l'anxiété}

Le graphique 1 illustre la relation entre les scores de détresse et d'anxiété. On constate que plus un répondant affirme ressentir de la détresse, plus il ressent également de l'anxiété et, inversement, moins il ressent de la détresse et moins il ressent de l'anxiété. La corrélation entre ces deux échelles de mesure est de $.62(p<.000)$.

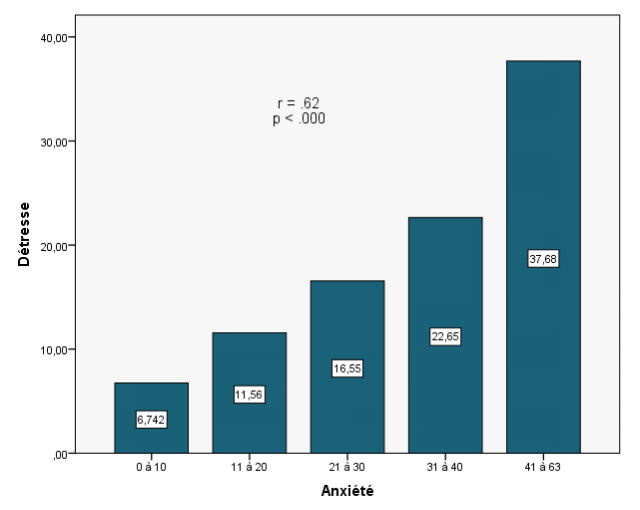

Graphique 1 : Relation entre le score total de détresse et le score total d'anxiété 


\section{Relation entre détresse, anxiété et utilisation des services d'aide}

De plus, les résultats indiquent qu'il y a une corrélation significative de .17 entre l'utilisation des ressources d'aide à l'interne et le score de détresse et de .19 avec l'échelle d'anxiété. Entre outre, la corrélation entre l'utilisation des ressources d'aide à l'externe et la détresse est de .24 et de .34 avec l'anxiété. En bref, plus on ressent de détresse ou d'anxiété, plus on utilise les services d'aide à l'interne, tout comme à l'externe.

\section{Préjugés à l'égard de la santé mentale}

Les préjugés à l'égard de la santé mentale sont apparus, lors d'une utilisation préliminaire, comme un frein important à l'utilisation des ressources d'aide. Afin de mesurer le niveau de préjugés, une échelle de mesure composée de 19 énoncés a été élaborée. Les répondants avaient à indiquer leur niveau d'accord ou de désaccord avec les énoncés selon une échelle variant de 1 à 4, 1 étant "Tout à fait en désaccord », 2 «En désaccord », 3 «En accord » et 4 « Tout à fait en accord ». Pour éviter les réponses stéréotypées, 8 des 19 énoncés ont été inversés de manière à mesurer l'absence de préjugé plutôt que sa présence. La moyenne obtenue à cette échelle est de 34,4 sur un total de 76 . Ce résultat suggère un niveau «Moyen faible » de préjugés chez les répondants. La mesure de l'alpha de Cronbach donne .79 pour les 19 éléments qui mesurent notre construit, ce qui indique à nouveau une cohérence interne relativement forte.

Tableau 4 Préjugés en santé mentale selon leur ordre d'importance

\begin{tabular}{|c|c|}
\hline & $\%$ \\
\hline $\begin{array}{l}\text { Au cours de leur vie, rares sont les personnes qui éprouvent des troubles d'adaptation } \\
\text { liés au stress }\end{array}$ & 47,6 \\
\hline Si on me disait atteint d'un problème de santé mentale, je ne l'accepterais pas & 33,6 \\
\hline $\begin{array}{c}\text { Si j'avais des problèmes personnels, je les garderais pour moi. Je n'en parlerais pas à } \\
\text { personne }\end{array}$ & 32,7 \\
\hline $\begin{array}{l}\text { Un environnement familial chaleureux et aimant immunise l'enfant contre des } \\
\text { problèmes de santé mentale }\end{array}$ & 31,3 \\
\hline Je peux avoir de l'influence sur la santé mentale de mon entourage & 25,9 \\
\hline Les maladies mentales peuvent être traitées efficacement & 25 \\
\hline $\begin{array}{l}\text { Il est probable que je puisse souffrir, au cours de ma vie, d'un problème de santé } \\
\text { mentale }\end{array}$ & 23,1 \\
\hline Consulter une ressource d'aide pourrait contribuer à mon épanouissement personnel & 21,5 \\
\hline $\begin{array}{c}\text { Au cours de l'Histoire, les sociétés humaines font face à la maladie mentale depuis } \\
\text { environ deux siècles seulement }\end{array}$ & 21,2 \\
\hline Consulter une ressource d'aide est un signe de faiblesse personnelle & 15,3 \\
\hline $\begin{array}{l}\text { Les personnes atteintes d'un problème de santé mentale manquent de volonté pour } \\
\text { s'en sortir }\end{array}$ & 14,7 \\
\hline $\begin{array}{l}\text { Il y a beaucoup moins à espérer de la vie après avoir été atteint d'un problème de santé } \\
\text { mentale }\end{array}$ & 13,9 \\
\hline $\begin{array}{l}\text { Les personnes atteintes d'un problème de santé mentale peuvent être aux études ou } \\
\text { sur le marché du travail }\end{array}$ & 12,8 \\
\hline $\begin{array}{l}\text { Les personnes qui consultent une ressource d'aide ne sont pas nécessairement } \\
\text { atteintes d'un problème de santé mentale. }\end{array}$ & 12,3 \\
\hline
\end{tabular}




\begin{tabular}{|c|c|}
\hline $\begin{array}{c}\text { Les gens atteints d'un problème de santé mentale sont plaignards, défaitistes et } \\
\text { incapables d'apprécier les beautés de la vie }\end{array}$ & 12,2 \\
\hline Les enfants peuvent être atteints d'un problème de santé mentale & 11,8 \\
\hline La maladie mentale apparaît rarement à l'adolescence et dans la jeune vie adulte & 10,4 \\
\hline Les personnes atteintes de maladie mentale sont violentes et dangereuses & 9,8 \\
\hline $\begin{array}{c}\text { Les maladies mentales touchent surtout des personnes âgées, pauvres et avec un } \\
\text { niveau d'instruction peu élevé }\end{array}$ & 7,3 \\
\hline
\end{tabular}

Le tableau 4, présente la répartition en pourcentage des préjugés en santé mentale selon leur ordre d'importance. Afin d'obtenir ces résultats nous avons additionné les réponses « En accord » et « Tout à fait en accord " sur chacun des énoncés. Les résultats du tableau montrent que 47,6\% des répondants affirment être en accord partiel ou total avec l'énoncé exprimant que " Au cours de leur vie, rares sont les personnes qui éprouveront des troubles d'adaptation liés au stress ". Également, près du tiers des répondants indiquent que "Si on me disait atteint d'un problème de santé mentale, je ne l'accepterais pas » (33,6\%), "Si j'avais des problèmes personnels, je les garderais pour moi. Je n'en parlerais pas à personne » $(32,7 \%)$ et "Un environnement familial chaleureux et aimant immunise l'enfant contre des problèmes de santé mentale " (31,3\%). Par ailleurs, moins de $10 \%$ de notre population pensent que "La maladie mentale apparaît rarement à l'adolescence et dans la jeune vie adulte ", "Les personnes atteintes de maladie mentale sont violentes et dangereuses " et "Les maladies mentales touchent surtout des personnes âgées, pauvres et avec un niveau d'instruction peu élevé ». De façon surprenante, seulement 1 répondant sur $4(25,9 \%)$ pense qu'il peut avoir de l'influence sur la santé mentale des personnes de son entourage et 1 répondant sur 6 (15,3\%) pense que « Consulter une ressource d'aide est un signe de faiblesse personnelle ».

\section{Préjugés et utilisation des ressources d'aide}

Les résultats indiquent quelques relations significatives entre les préjugés et l'utilisation des ressources d'aide à l'interne et à l'externe. Bien que les corrélations soient faibles la relation est néanmoins significative et va dans le sens de notre hypothèse selon laquelle les préjugés sont un frein à l'utilisation des ressources d'aide. Ainsi, plus le score de préjugés est élevé, moins on consulte de ressources d'aide $(r=-.15, p<.000)$.

Parmi les préjugés cités dans l'enquête, deux ressortent davantage comme réduisant la probabilité d'une utilisation d'une ressource d'aide soit "Consulter une ressource d'aide ne pourrait pas contribuer à mon épanouissement personnel » et «ll est peu probable que je puisse souffrir au cours de ma vie d'un problème de santé mentale ».

\section{Relation entre les préjugés la détresse et l'anxiété}

Cette étude permet d'établir des relations significatives, bien que faibles, entre le score total de préjugés à l'égard de la santé mentale et les scores totaux obtenus aux échelles de détresse et d'anxiété. Également, certains préjugés comme "consulter une ressource d'aide est un signe de faiblesse personnelle " et "il y a beaucoup moins à espérer de la vie après avoir été atteint d'un problème de santé mentale" sont corrélés significativement avec les pensées suicidaires. En conséquence, les préjugés nuisent à l'utilisation des ressources d'aide et particulièrement chez les personnes qui présentent des pensées suicidaires. 


\section{Déterminants des problèmes socioaffectifs}

En se basant sur les fréquences, la force des inter-corrélations entre les facteurs ci-étudiés et divers tests-t entre les répondants qui se situent dans le haut et le bas de nos échelles de mesure, quatre facteurs apparaissent jouer un rôle important dans la compréhension de la détresse et de l'anxiété chez les étudiants du collégial soit: les conflits familiaux, la pression liée à la performance scolaire, l'intimidation et les pensées suicidaires.

\section{- Conflits familiaux}

Rappelons que 62,2 \% des répondants disent avoir vécu de la détresse en lien avec les conflits familiaux et que de ce nombre 29,3\% en ont vécu «Beaucoup 》 ou "Énormément 》. De plus, les résultats indiquent une corrélation de .62 entre les conflits familiaux et le score de détresse et de .44 avec l'anxiété. En définitive, plus on vit de conflits familiaux, plus on ressent de la détresse et de l'anxiété.

\section{- Pression liée à la performance scolaire}

Rappelons aussi que $61,4 \%$ des répondants disent avoir ressenti de la détresse à l'égard de la pression liée à la performance scolaire et que de ce nombre 33,3\% disent en avoir ressenti «Beaucoup » ou «Énormément ». De plus, les résultats indiquent une corrélation de .62 entre la pression liée à la performance scolaire et le score de détresse et de .56 avec l'anxiété. En définitive, plus on vit de pression scolaire, plus on ressent de la détresse et de l'anxiété.

\section{- Intimidation}

Signalons également que $19 \%$ des répondants disent être victime d'intimidation au moment où ils ont répondu au questionnaire et de ce nombre 11,9\% disent l'être "Beaucoup " ou «Énormément ». De plus, l'intimidation présente des corrélations significatives avec plusieurs facteurs abordés dans l'enquête, dont le rejet (.81), la détresse (.77), l'agression ou la violence (.72), le conflit culturel (.72) et le handicap physique (.71).

Ajoutons que les résultats indiquent une corrélation de .42 entre l'intimidation et les pensées suicidaires. L'augmentation des pensées suicidaires est proportionnelle à la fréquence de l'intimidation. De plus, on constate que les pensées suicidaires chez ceux qui éprouvent fortement de l'intimidation sont 5 fois plus fréquentes que chez ceux qui n'en vivent pas.

\section{- Pensées suicidaires}

Précisons finalement que 18,3\% des répondants (2270) affirment avoir des pensées suicidaires et que parmi eux, 7,3\% des répondants (891) disent en avoir « Souvent » ou « Tout le temps ». L'ampleur de ces chiffres devrait inciter à étudier ce phénomène plus en profondeur.

Les pensées suicidaires sont corrélées surtout au score de détresse (.60), aux conflits culturels (.60), au score d'anxiété (.59) et à un handicap physique (.59).

\section{Discussion}

Insistons sur l'importance des résultats découlant de l'enquête. Rappelons que 12208 étudiants provenant de huit cégeps différents répartis sur plusieurs régions du Québec ont répondu à l'enquête, ce qui donne un portrait intéressant des difficultés socioaffectives vécues au collégial. Conduite à 
l'ensemble du réseau collégial, une telle enquête conduirait à dresser un tableau inédit des problèmes socioaffectifs de la jeunesse québécoise.

II ressort de cette étude, que plusieurs lacunes existent dans l'offre des ressources d'aide pour répondre aux besoins des étudiants des cégeps. L'enquête montre que plusieurs ressources comme les travailleurs sociaux, les psychologues et les éducateurs spécialisés, entre autres, ne sont présents que dans quelques cégeps, alors que ce sont eux qui pourraient faire face aux problèmes de détresse, d'anxiété, d'intimidation et de pensées suicidaires. En leur absence, même s'ils sont bien intentionnés et que ce mandat ne relève pas de leurs tâches professionnelles, ce sont les professeurs, les API, les $\mathrm{CO}$ et les agents de sécurité qui doivent intervenir alors qu'ils n'ont pas nécessairement la formation professionnelle requise pour ce genre d'intervention. Dans le contexte des coupures budgétaires actuelles en éducation, peut-on être à l'aise avec l'idée que la seule issue pour les $15 \%$ des étudiants en situation de détresse et d'anxiété élevées soit le recours aux ressources d'aide à l'externe ?

\section{Conclusions}

Au terme de cette enquête, on peut conclure que les ressources d'aide à l'interne sont utilisées par les trois quarts des répondants et $85 \%$ disent les utiliser à l'externe.

Les étudiants qui utilisent les plus les ressources à l'interne sont également ceux qui les utilisent le plus à l'externe et les filles utilisent de telles ressources deux fois plus que les garçons.

La satisfaction et la curiosité sont les principaux motifs invoqués pour l'utilisation des ressources d'aide à l'interne, néanmoins, la souffrance constitue un motif important d'utilisation.

La détresse et l'anxiété sont parmi les mesures qui nous ont permis d'évaluer l'ampleur des besoins en services socioaffectifs des répondants. À la lumière des résultats, une grande conclusion s'impose soit la nécessité d'offrir des services d'aide aux étudiants qui éprouvent des difficultés personnelles.

Les conflits familiaux, la pression liée à la performance scolaire, l'intimidation et les pensées suicidaires sont des facteurs importants dans la compréhension des problèmes socioaffectifs des étudiants au collégial

Le niveau de préjugés à l'égard de la santé mentale chez les répondants est relativement « moyen ». II y a une relation significative, bien que faible, entre le score total de préjugés à l'égard de la santé mentale et le nombre d'utilisation des ressources d'aide à l'interne comme à l'externe. En conséquence, les préjugés constituent un frein à l'utilisation des ressources d'aide et plus particulièrement chez les personnes qui ont des pensées suicidaires.

Marc-André Gosselin, M. Ps.

Professeur de psychologie, Collège de Bois-de-Boulogne

Robert Ducharme, B. Ps.

Professeur de psychologie retraité, Cégep de St-Jérôme 


\section{RÉFÉRENCES}

American Psychiatric Association (2013). Diagnostic statistical manual (DSM-V),Washington (DC), American Psychiatric Association.

Beck, A.T., et R.A. Steer (1992). «Relationship between the BAI and Hamilton Anxiety Rating Scale with anxious outpatients », Journal of Anxiety Disorders, vol. 5, n 3, p. 213-223.

Daddona, M.F. (2011). «Peer Educators Responding to Students with Mental Health Issues », New Directions for Student Services, $n^{\circ} 133,2011$, p. 29-39.

Fier, S.M., et L.G. Brzezinski (2010). «Facilitating the High School-to-College Transition for Students with Psychiatric Disabilities: Information and Strategies for School Counselors », Journal of School Counseling, vol. 8, $\mathrm{n}^{\circ} 10$.

Gosselin, M.-A., et R. Ducharme (2015). Résultats à l'enquête concernant les besoins des étudiants à l'égard des services d'aide psychoaffectifs dans le réseau collégial à la session d'automne 2014, Webinaire, CAPRES, octobre 2015.

Gosselin, M.-A., et R. Ducharme (2014). Besoins psychosociaux des étudiants et l'utilisation des services d'aide au collège de Bois-de-Boulogne à la session automne 2013, Montréal, Collège de Bois-de-Boulogne.

Gosselin, M.-A., et R. Ducharme (2016). Besoins psychosociaux des étudiants et l'utilisation des services d'aide dans le réseau collégial à la session d'automne 2014, Montréal, Collège de Bois-deBoulogne.

Holmes, T.H., et R.H. Rahe (1967). « The social readjustment rating scale », Journal of Psychosomatic Research, vol. 11, n², p. 213-218.

Lesage, A., F. Bernèche et M. Bordeleau (2010). Étude sur la santé mentale et le bien-être des adultes québécois : une synthèse pour soutenir l'action. Enquête sur la santé dans les collectivités canadiennes (cycle 1.2), Québec, Institut de la statistique du Québec, 104 p.

Villatte, A., D. Marcotte, et A. Potvin (2015). "Ces étudiants à risque de dépression », Le Sociographe, $\mathrm{n}^{\circ} 51$, p. 65-75. 